\title{
On the distribution of the $\mathbf{F}_{p}$-points on an affine curve in $r$ dimensions
}

\author{
by \\ Cristian Cobeli (Bucureşti) and \\ Alexandru Zaharescu (Bucureşti and Urbana, IL)
}

1. Introduction and statement of results. Let $p$ be a prime number, $\overline{\mathbf{F}}_{p}$ the algebraic closure of $\mathbf{F}_{p}=\mathbb{Z} / p \mathbb{Z}$ and let $\mathcal{C}$ be an irreducible curve of degree $d$ in an affine space $\mathbb{A}^{r}\left(\overline{\mathbf{F}}_{p}\right)$. We assume in the following that $\mathcal{C}$ is not contained in any hyperplane and that it is defined over $\mathbf{F}_{p}$. Our object in this paper is to study the distribution of the set $\mathcal{C}\left(\mathbf{F}_{p}\right)$ of $\mathbf{F}_{p}$-points on $\mathcal{C}$. We are interested in obtaining asymptotic results as $p$ goes to infinity, while $r$ is fixed, $d$ is bounded and $\mathcal{C}$ is as above. In particular we would like to understand the distribution of distances between the coordinates of a point $\mathbf{x}=\left(x_{1}, \ldots, x_{r}\right) \in \mathbf{F}_{p}^{r}$ which moves along the curve. Our original motivation for investigating these distances came from the problem of the distribution of $|a-\bar{a}|$, where $a, \bar{a}$ run over the set $\{1, \ldots, p-1\}$ such that $a \bar{a} \equiv 1(\bmod p)$. This problem was solved by Wenpeng Zhang [4] who proved that for any integer $n \geq 2$ and any $0<\delta \leq 1$,

$$
\begin{aligned}
\mid\{a: 1 \leq a \leq n-1,(a, n)= & 1,|a-\bar{a}|<\delta n\} \mid \\
& =\delta(2-\delta) \varphi(n)+O\left(n^{1 / 2} d^{2}(n) \log ^{3} n\right),
\end{aligned}
$$

where $\varphi(n)$ is the Euler function and $d(n)$ denotes the number of divisors of $n$. In [5] Zhiyong Zheng investigated the same problem, with $(a, \bar{a})$ replaced by a pair $(x, y)$ satisfying a more general congruence. Precisely, let $p$ be a prime number and let $f(x, y)$ be a polynomial with integer coefficients of total degree $d \geq 2$, absolutely irreducible modulo $p$. Then it is proved in [5] that for any $0<\delta \leq 1$,

$$
\begin{aligned}
\mid\left\{(x, y) \in \mathbb{Z}^{2}: 0 \leq x, y<p, f(x, y)\right. & \equiv 0(\bmod p),|x-y|<\delta p\} \mid \\
& =\delta(2-\delta) p+O_{d}\left(p^{1 / 2} \log ^{2} p\right) .
\end{aligned}
$$

2000 Mathematics Subject Classification: Primary 11T99. 
Returning to our context, we fix an $r \geq 2$, then choose a large prime number $p$ and a curve $\mathcal{C}$ in $\mathbb{A}^{r}\left(\overline{\mathbf{F}}_{p}\right)$ as before. We view $\mathcal{C}\left(\mathbf{F}_{p}\right)$ as sitting in the torus $\mathbb{T}^{r}=\mathbb{R}^{r} / \mathbb{Z}^{r}$. Precisely, one has a natural injection $\mathbf{F}_{p} \rightarrow \mathbb{T}=\mathbb{R} / \mathbb{Z}$ defined as follows. Given $x \in \mathbf{F}_{p}$, choose a representative $m$ of $x$ in $\mathbb{Z}$ and then project $m / p \in \mathbb{R}$ to its image $t=t(x)$ in $\mathbb{T}$. Note that $t(x)$ does not depend on the choice of $m$. Then $\mathbf{F}_{p}^{r}$ injects in $\mathbb{T}^{r}$, a point $\mathbf{x}=\left(x_{1}, \ldots, x_{r}\right) \in$ $\mathbf{F}_{p}^{r}$ being sent to $t(\mathbf{x})=\left(t\left(x_{1}\right), \ldots, t\left(x_{r}\right)\right) \in \mathbb{T}^{r}$. To study the distribution of $\mathcal{C}\left(\mathbf{F}_{p}\right)$ in $\mathbb{T}^{r}$, consider the probability measure $\mu_{r, p, \mathcal{C}}$ on $\mathbb{T}^{r}$ defined by

$$
\mu_{r, p, \mathcal{C}}=\frac{1}{\left|\mathcal{C}\left(\mathbf{F}_{p}\right)\right|} \sum_{\mathbf{x} \in \mathcal{C}\left(\mathbf{F}_{p}\right)} \delta_{t(\mathbf{x})},
$$

where $\delta_{t(\mathbf{x})}$ is a unit point delta mass at $t(\mathbf{x})$. The first question one would ask about these measures is whether they converge to a certain measure on $\mathbb{T}^{r}$ as $p \rightarrow \infty$ and $\mathcal{C}$ is as before, say of bounded degree $d$. The answer is that they weakly converge to the (normalized) Haar measure $\mu$ on $\mathbb{T}^{r}$. Next, we would like to know how fast $\mu_{r, p, \mathcal{C}}(\Omega)$ approaches $\mu(\Omega)$ for a given nice domain $\Omega$ in $\mathbb{T}^{r}$, so that one could produce quantitative results for any large prime number $p$. We prove the following

Theorem 1. Let $r \geq 2$ be an integer and $\Omega$ a domain in $\mathbb{T}^{r}$ with piecewise smooth boundary. Then for any prime $p$ and any irreducible curve $\mathcal{C}$ of degree d in $\mathbb{A}^{r}\left(\overline{\mathbf{F}}_{p}\right)$, defined over $\mathbf{F}_{p}$ and not contained in any hyperplane,

$$
\mu_{r, p, \mathcal{C}}(\Omega)=\mu(\Omega)+O_{r, d, \Omega}\left(p^{-1 /(2(r+1))} \log ^{r /(r+1)} p\right) .
$$

Next, we look at the distances between the coordinates $x_{1}, \ldots, x_{r}$ of a point $\mathbf{x} \in \mathcal{C}\left(\mathbf{F}_{p}\right)$. Consider the concrete problem of finding, for a given $\boldsymbol{\delta}=\left(\delta_{1}, \ldots, \delta_{r-1}\right) \in \mathbb{R}^{r-1}$ with $0<\delta_{1}, \ldots, \delta_{r-1} \leq 1$, the proportion $\varrho_{r, p, \mathcal{C}, \boldsymbol{\delta}}$ of points $\mathbf{x} \in \mathcal{C}\left(\mathbf{F}_{p}\right)$ for which

$$
\left|t\left(x_{1}\right)-t\left(x_{r}\right)\right| \leq \delta_{1}, \quad\left|t\left(x_{2}\right)-t\left(x_{r}\right)\right| \leq \delta_{2}, \ldots,\left|t\left(x_{r-1}\right)-t\left(x_{r}\right)\right| \leq \delta_{r-1} .
$$

The map from $\mathbb{R}^{r}$ to $\mathbb{R}^{r-1}$ given by $\left(y_{1}, \ldots, y_{r}\right) \mapsto\left(y_{1}-y_{r}, \ldots, y_{r-1}-y_{r}\right)$ sends $\mathbb{Z}^{r}$ to $\mathbb{Z}^{r-1}$, and so it induces a map, call it $\psi$, from $\mathbb{T}^{r}$ to $\mathbb{T}^{r-1}$. Note that $\psi$ is additive and it preserves the Haar measure:

$$
\mu(U)=\mu\left(\psi^{-1}(U)\right)
$$

for any open subset $U$ of $\mathbb{T}^{r-1}$, where we denoted by $\mu$ the Haar measure on both $\mathbb{T}^{r}$ and $\mathbb{T}^{r-1}$. Let $U_{\delta}$ be the image in $\mathbb{T}^{r-1}$ of the box $\left[-\delta_{1}, \delta_{1}\right] \times$ $\ldots \times\left[-\delta_{r-1}, \delta_{r-1}\right] \subset \mathbb{R}^{r-1}$. Assume $0<\delta_{1}, \ldots, \delta_{r-1} \leq 1 / 2$, then $\mu\left(U_{\delta}\right)=$ $2^{r-1} \delta_{1} \ldots \delta_{r-1}$. By the definition of $\mu_{r, p, \mathcal{C}}, \psi$ and $\varrho_{r, p, \mathcal{C}, \delta}$ one sees that

$$
\varrho_{r, p, \mathcal{C}, \delta}=\mu_{r, p, \mathcal{C}}\left(\psi^{-1}\left(U_{\delta}\right)\right) .
$$

Using (4), (3) and Theorem 1 with $\Omega=\psi^{-1}\left(U_{\delta}\right)$ we obtain

$$
\varrho_{r, p, \mathcal{C}, \boldsymbol{\delta}}=2^{r-1} \delta_{1} \ldots \delta_{r-1}+O_{r, d, \delta}\left(p^{-1 /(2(r+1))} \log ^{r /(r+1)} p\right) .
$$


A more accurate version of (5) is stated in Corollary 1 from Section 4 below. In case $r=2, \delta_{1}=\delta$, from (5) one derives

$$
\varrho_{r, p, \mathcal{C}, \boldsymbol{\delta}} \sim 2 \delta \quad \text { as } p \rightarrow \infty .
$$

The reader noticed that this is different from the asymptotic result which follows from (2). The reason for this comes from the way the distance was defined: in (5) the distances are computed on the torus $\mathbb{T}$ while in (2) the set $\mathcal{C}\left(\mathbf{F}_{p}\right)$ is injected in a Euclidean space. Precisely, the points $(x, y) \in \mathbb{Z}^{2}$, $0 \leq x, y<p,(x, y)(\bmod p) \in \mathcal{C}$ for which $|x-y-p|<\delta p$ or $|x-y+p|$ $<\delta p$ do contribute to $\varrho_{r, p, \mathcal{C}, \boldsymbol{\delta}}$, and they account for the difference $\delta^{2}$ in the two asymptotic results. Actually one can recover a result of type (2) from Theorem 1 above, as follows. Fix a point $\mathbf{u} \in \mathbb{T}^{r}$ and choose a representative $\mathbf{v}=\left(v_{1}, \ldots, v_{r}\right)$ of $\mathbf{u}$ in $\mathbb{R}^{r}$. Any $\mathbf{t}=\left(t_{1}, \ldots, t_{r}\right) \in \mathbb{T}^{r}$ has a unique representative $\left(v_{1}+y_{1}, \ldots, v_{r}+y_{r}\right) \in \mathbb{R}^{r}$ with $0 \leq y_{1}, \ldots, y_{r}<1$. We define the distances between the components of $\mathbf{t}$ with respect to $\mathbf{v}$, by

$$
\left|t_{i}-t_{j}\right|_{\mathbf{v}}=\left|y_{i}-y_{j}\right|, \quad 1 \leq i, j \leq r .
$$

These distances depend on $\mathbf{u}$ but not on the choice of $\mathbf{v}$, so we denote them by $\left|t_{i}-t_{j}\right|_{\mathbf{u}}$. Given a point $\mathbf{t} \in \mathbb{T}^{r}$ and two of its components $t_{i}, t_{j} \in \mathbb{T}$, there are two $\operatorname{arcs}$ in $\mathbb{T}$ which join the points $t_{i}$ and $t_{j}$. For any $\mathbf{u} \in \mathbb{T}^{r}$ the distance $\left|t_{i}-t_{j}\right|_{\mathbf{u}}$ equals the length of one of these two arcs. Thus in some sense working with distances with respect to a fixed point $\mathbf{u} \in \mathbb{T}^{r}$ gives us a coherent way of choosing between the above two arcs associated to any pair $\left(t_{i}, t_{j}\right)$, as $\mathbf{t}$ runs over $\mathbb{T}^{r}$. We now consider for a given $\mathbf{u} \in \mathbb{T}^{r}$ and a given $\boldsymbol{\delta}=\left(\delta_{1}, \ldots, \delta_{r-1}\right) \in \mathbb{R}^{r-1}$ with $0<\delta_{1}, \ldots, \delta_{r-1} \leq 1$, the proportion $\eta_{r, p, \mathcal{C}, \mathbf{u}, \delta}$ of points $\mathbf{x}=\left(x_{1}, \ldots, x_{r}\right) \in \mathcal{C}\left(\mathbf{F}_{p}\right)$ for which

$$
\left|t\left(x_{1}\right)-t\left(x_{r}\right)\right|_{\mathbf{u}} \leq \delta_{1}, \quad \ldots, \quad\left|t\left(x_{r-1}\right)-t\left(x_{r}\right)\right|_{\mathbf{u}} \leq \delta_{r-1} .
$$

We remark that $\eta_{r, p, \mathcal{C}, \mathbf{u}, \delta}$ does depend on $\mathbf{u}$. However, the fact that changing $\mathbf{u}$, that is, changing the lifting of $\mathbb{T}^{r}$ in $\mathbb{R}^{r}$ does not affect the Haar measure which is invariant under translations, together with the fact that $\mu_{r, p, \mathcal{C}}$ approaches the Haar measure as $p \rightarrow \infty$, make $\eta_{r, p, \mathcal{C}, \mathbf{u}, \delta}$ converge to a limit which is independent of $\mathbf{u}$. To state our result, we introduce the following function $h:[0,1] \times[0,1] \rightarrow[0,1]$. For $0 \leq z<1 / 2$ we define

$$
h(y, z)= \begin{cases}y+z & \text { if } 0 \leq y<z \\ 2 z & \text { if } z \leq y<1-z \\ 1-y+z & \text { if } 1-z \leq y \leq 1\end{cases}
$$

For $1 / 2 \leq z \leq 1$ we let

$$
h(y, z)= \begin{cases}y+z & \text { if } 0 \leq y<1-z \\ 1 & \text { if } 1-z \leq y<z \\ 1-y+z & \text { if } z \leq y \leq 1\end{cases}
$$


Next, for any $0<\delta_{1}, \ldots, \delta_{r-1} \leq 1$ we set

$$
c\left(\delta_{1}, \ldots, \delta_{r-1}\right)=\int_{0}^{1} \prod_{j=1}^{r-1} h\left(y, \delta_{j}\right) d y .
$$

Then we prove the following result.

Theorem 2. Let $r \geq 2$ be an integer, $\mathbf{u} \in \mathbb{T}^{r}, \boldsymbol{\delta}=\left(\delta_{1}, \ldots, \delta_{r-1}\right) \in \mathbb{R}^{r-1}$ with $0<\delta_{1}, \ldots, \delta_{r-1} \leq 1, p$ a prime number and $\mathcal{C}$ an irreducible curve of degree $d$ in $\mathbb{A}^{r}\left(\overline{\mathbf{F}}_{p}\right)$, defined over $\mathbf{F}_{p}$ and not contained in any hyperplane. Then

$$
\eta_{r, p, \mathcal{C}, \mathbf{u}, \delta}=c\left(\delta_{1}, \ldots, \delta_{r-1}\right)+O_{r, d, \mathbf{u}, \delta}\left(p^{-1 /(2(r+1))} \log ^{r /(r+1)} p\right) .
$$

In particular, when $r=2$ one has

$$
c(\delta)=\int_{0}^{1} h(y, \delta) d y=2 \delta-\delta^{2}
$$

which agrees with (2).

Acknowledgements. The authors are grateful to Andrew Granville for suggesting the problem which led to Theorem 1 above. They are also grateful to the referee whose suggestions improved the presentation of the paper.

2. Proof of Theorem 1. Let $r, p, \mathcal{C}$ and $\Omega$ be as in the statement of the theorem. We split the torus $\mathbb{T}^{r}$ in little cubes with edge length $1 / T$, where $T$ is a positive integer. As we shall see later, the optimal choice for $T$ in this proof is $T=\left[p^{1 /(2(r+1))} \log ^{-r /(r+1)} p\right]$. For each such cube $\mathbf{J}$ one has $\mu(\mathbf{J})=T^{-r}$. We denote by $\mathcal{D}(T)$ the union of those cubes contained in $\Omega$ and by $E(T)$ the union of those cubes which have a nonempty intersection with $\Omega$. Therefore

$$
\mathcal{D}(T) \subseteq \Omega \subseteq E(T) .
$$

Now fix an arbitrary such cube $\mathbf{J}$ and estimate the number $N(\mathbf{J})$ of points $\mathbf{x} \in \mathcal{C}\left(\mathbf{F}_{p}\right)$ for which $t(\mathbf{x}) \in \mathbf{J}$. Since $\mathbf{J}$ is a cube, there are subsets $J_{1}, \ldots, J_{r}$ of $\mathbf{F}_{p}$ of the form $J_{j}=\left\{a_{j}+1, a_{j}+2, \ldots, a_{j}+b_{j}\right\}, 1 \leq j \leq r$, such that a point $\mathbf{x} \in \mathbf{F}_{p}^{r}$ lies in $J_{1} \times \ldots \times J_{r}$ if and only if $t(\mathbf{x}) \in \mathbf{J}$. The number of elements of $J_{j}$ is

$$
\#\left(J_{j}\right)=p / T+O(1), \quad 1 \leq j \leq r .
$$

One has

$$
N(\mathbf{J})=\sum_{\mathbf{x} \in \mathcal{C}\left(\mathbf{F}_{p}\right)} \chi_{\mathcal{J}_{1}}\left(x_{1}\right) \ldots \chi_{\mathcal{J}_{r}}\left(x_{r}\right)
$$


where $\chi_{\mathcal{J}}(x)$ is the characteristic function of the interval $\mathcal{J}$. An analytic expression for $\chi_{\mathcal{J}}(x)$ with $x \in \mathbf{F}_{p}$ is given by

$$
\chi_{\mathcal{J}}(x)=\sum_{y \in \mathcal{J}} \frac{1}{p} \sum_{k(\bmod p)} e_{p}(k(x-y)),
$$

where $e_{p}(x)=e^{2 \pi i x / p}$. Using (8) in (7) and changing the order of summation, we obtain

$$
N(\mathbf{J})=\frac{1}{p^{r}} \sum_{k_{1}(\bmod p)} \ldots \sum_{k_{r}(\bmod p)} \prod_{j=1}^{r}\left(\sum_{y_{j} \in \mathcal{J}_{j}} e_{p}\left(-k_{j} y_{j}\right)\right) S_{\mathbf{k}}(\mathbf{x}),
$$

where $\mathbf{k}=\left(k_{1}, \ldots, k_{r}\right)$ and

$$
S_{\mathbf{k}}(\mathbf{x})=S_{\mathbf{k}, p, r, \mathcal{C}}(\mathbf{x})=\sum_{\mathbf{x} \in \mathcal{C}\left(\mathbf{F}_{p}\right)} e_{p}\left(k_{1} x_{1}+\ldots+k_{r} x_{r}\right) .
$$

Since by hypothesis $\mathcal{C}$ is not contained in any hyperplane it follows that the linear form $k_{1} x_{1}+\ldots+k_{r} x_{r}$ is constant along $\mathcal{C}$ if and only if $k_{1}=\ldots=k_{r}$ $=0$. This suggests separating the sum of the terms with $k_{1}=\ldots=k_{r}=0$ and we will see that they give the main contribution in (9). It equals

$$
M=\frac{1}{p^{r}}\left(\prod_{j=1}^{r}\left|\mathcal{J}_{j}\right|\right) \sum_{\mathbf{x} \in \mathcal{C}\left(\mathbf{F}_{p}\right)} 1=\frac{\left|\mathcal{C}\left(\mathbf{F}_{p}\right)\right|}{T^{r}}\left(1+O_{r}\left(\frac{T}{p}\right)\right) .
$$

By the Riemann Hypothesis for curves over finite fields (Weil [3]) we know that

$$
\left|\mathcal{C}\left(\mathbf{F}_{p}\right)\right|=p+O_{r, d}(\sqrt{p}) .
$$

In what follows we assume that $T \leq \sqrt{p}$. Then we have

$$
M=\frac{p}{T^{r}}\left(1+O_{r, d}\left(\frac{1}{\sqrt{p}}\right)\right) .
$$

The remainder is

$$
E=\frac{1}{p^{r}} \sum_{\mathbf{k}(\bmod p)}^{\prime} \prod_{j=1}^{r}\left(\sum_{y_{j} \in \mathcal{J}_{j}} e_{p}\left(-k_{j} y_{j}\right)\right) S_{\mathbf{k}}(\mathbf{x}),
$$

where the prime means that the terms with $k_{1}=\ldots=k_{r}=0$ are excluded from the summation. Each of the factors of the product over $j(1 \leq j \leq r)$ is a geometric progression and can be estimated accurately. Indeed, we have

$$
\begin{aligned}
\left|\sum_{y_{j} \in \mathcal{J}_{j}} e_{p}\left(-k_{j} y_{j}\right)\right| & \leq \min \left\{\left|\mathcal{J}_{j}\right|, \frac{2}{\left|1-e_{p}\left(k_{j}\right)\right|}\right\} \leq \min \left\{\left|\mathcal{J}_{j}\right|, \frac{1}{\left|\sin \frac{\pi k_{j}}{p}\right|}\right\} \\
& \leq \min \left\{\left|\mathcal{J}_{j}\right|, \frac{1}{2\left\|\frac{k_{j}}{p}\right\|}\right\},
\end{aligned}
$$


where $\|\cdot\|$ denotes the distance to the nearest integer. For each $\mathbf{k} \neq \mathbf{0}$ our hypotheses on $\mathcal{C}$ allow us to apply the Bombieri-Weil inequality (see $[1$, Theorem 6]), which gives $S_{\mathbf{k}}(\mathbf{x})=O_{r, d}\left(p^{1 / 2}\right)$. Assuming, as we can, that in the summation over $\mathbf{k}$ in the definition of $E$ one has $\left|k_{j}\right| \leq(p-1) / 2$ for $1 \leq j \leq r$, we obtain

$$
\begin{aligned}
|E| & \leq \frac{1}{p^{r}} \sum_{\mathbf{k}(\bmod p)}^{\prime} \prod_{j=1}^{r}\left(\min \left\{\frac{p}{T}, \frac{p}{\left|k_{j}\right|}\right\}\right)\left|S_{\mathbf{k}}(\mathbf{x})\right| \\
& \ll_{r} \sum_{\mathbf{k}(\bmod p)}^{\prime} \frac{1}{T+\left|k_{1}\right|} \cdots \frac{1}{T+\left|k_{r}\right|}\left|S_{\mathbf{k}}(\mathbf{x})\right| .
\end{aligned}
$$

Consequently we deduce

$$
|E|=O_{r, d}\left(p^{1 / 2} \log ^{r} p\right) .
$$

By putting together (12) and (13) we obtain the required estimation for a cube:

$$
N(\mathbf{J})=p / T^{r}+O_{r, d}\left(p^{1 / 2} \log ^{r} p\right) .
$$

We know by the Lipschitz principle on the number of integer points in an $r$-dimensional domain (see Davenport [2]), applied in this case via our lifting of $\mathbb{T}^{r}$ in $\mathbb{R}^{r}$, that

$$
\mu(E(T) \backslash \mathcal{D}(T))=O_{r, \Omega}(1 / T) .
$$

That is, both $\mathcal{D}(T)$ and $E(T)$ are unions of $T^{r} \mu(\Omega)+O_{\Omega, r}\left(T^{r-1}\right)$ cubes with edge equal to $1 / T$. Using (14) for all these cubes one obtains

$$
\begin{aligned}
\mid\left\{\mathbf{x} \in \mathcal{C}\left(\mathbf{F}_{p}\right): t(\mathbf{x})\right. & \in \mathcal{D}(T)\} \mid \\
& =\left(p / T^{r}+O_{r, d}\left(p^{1 / 2} \log ^{r} p\right)\right)\left(T^{r} \mu(\Omega)+O_{\Omega, r}\left(T^{r-1}\right)\right) \\
& =p \mu(\Omega)+O_{r, d, \Omega}\left(T^{r} p^{1 / 2} \log ^{r} p\right)+O_{\Omega, r}(p / T)
\end{aligned}
$$

and similarly

$$
\left|\left\{\mathbf{x} \in \mathcal{C}\left(\mathbf{F}_{p}\right): t(\mathbf{x}) \in E(T)\right\}\right|=p \mu(\Omega)+O_{r, d, \Omega}\left(T^{r} p^{1 / 2} \log ^{r} p\right)+O_{\Omega, r}(p / T) .
$$

Therefore

$$
\left|\left\{\mathbf{x} \in \mathcal{C}\left(\mathbf{F}_{p}\right): t(\mathbf{x}) \in \Omega\right\}\right|=p \mu(\Omega)+O_{r, d, \Omega}\left(T^{r} p^{1 / 2} \log ^{r} p\right)+O_{\Omega, r}(p / T) .
$$

Since

$$
\mu_{r, p, \mathcal{C}}(\Omega)=\frac{\left|\left\{\mathbf{x} \in \mathcal{C}\left(\mathbf{F}_{p}\right): t(\mathbf{x}) \in \Omega\right\}\right|}{\left|\mathcal{C}\left(\mathbf{F}_{p}\right)\right|},
$$

from (15) and (11) it follows that

$$
\mu_{r, p, \mathcal{C}}(\Omega)=\mu(\Omega)+O_{r, d, \Omega}\left(T^{r} p^{-1 / 2} \log ^{r} p\right)+O_{r, d, \Omega}(1 / T) .
$$

We now choose

$$
T=\left[p^{1 /(2(r+1))} \log ^{-r /(r+1)} p\right]
$$


which gives

$$
\mu_{r, p, \mathcal{C}}(\Omega)=\mu(\Omega)+O_{r, d, \Omega}\left(p^{-1 /(2(r+1))} \log ^{r /(r+1)} p\right)
$$

and this completes the proof of Theorem 1 .

3. Proof of Theorem 2. Let $r, p, \mathcal{C}, \mathbf{u}$ and $\boldsymbol{\delta}$ be as in the statement of the theorem. Choose a representative $\mathbf{v}=\left(v_{1}, \ldots, v_{r}\right)$ of $\mathbf{u}$ in $\mathbb{R}^{r}$. Inside the box

$$
B=\left\{\left(v_{1}+y_{1}, \ldots, v_{r}+y_{r}\right): 0 \leq y_{1}, \ldots, y_{r}<1\right\}
$$

consider the region

$$
A=\left\{\left(v_{1}+y_{1}, \ldots, v_{r}+y_{r}\right) \in B:\left|y_{j}-y_{r}\right| \leq \delta_{j}, 1 \leq j \leq r-1\right\} .
$$

Let $\Omega$ be the image of $A$ in $\mathbb{T}^{r}$. The canonical map from $A$ to $\Omega$ is one-to-one and we have

$$
\operatorname{Vol}(A)=\mu(\Omega) .
$$

By the definition of $\eta_{r, p, \mathcal{C}, \mathbf{u}, \delta}$ we see that

$$
\eta_{r, p, \mathcal{C}, \mathbf{u}, \boldsymbol{\delta}}=\frac{\left|\left\{\mathbf{x} \in \mathcal{C}\left(\mathbf{F}_{p}\right): t(\mathbf{x}) \in \Omega\right\}\right|}{\left|\mathcal{C}\left(\mathbf{F}_{p}\right)\right|}=\mu_{r, p, \mathcal{C}}(\Omega) .
$$

From Theorem 1 and (17) we deduce

$$
\eta_{r, p, \mathcal{C}, \mathbf{u}, \boldsymbol{\delta}}=\operatorname{Vol}(A)+O_{r, d, \mathbf{u}, \boldsymbol{\delta}}\left(p^{-1 /(2(r+1))} \log ^{r /(r+1)} p\right) .
$$

It remains to compute $\operatorname{Vol}(A)$. Set

$$
z_{j}=\max \left\{0, y_{r}-\delta_{j}\right\}, \quad w_{j}=\min \left\{1, y_{r}+\delta_{j}\right\}, \quad 1 \leq j \leq r-1 .
$$

Then

$$
\operatorname{Vol}(A)=\int_{0}^{1} \int_{z_{r-1}}^{w_{r-1}} \ldots \int_{z_{1}}^{w_{1}} d y_{1} \ldots d y_{r}=\int_{0}^{1}\left(w_{1}-z_{1}\right) \ldots\left(w_{r-1}-z_{r-1}\right) d y_{r} .
$$

One checks that

$$
w_{j}-z_{j}=h\left(y_{r}, \delta_{j}\right), \quad 1 \leq j \leq r-1 .
$$

Hence $\operatorname{Vol}(A)=c\left(\delta_{1}, \ldots, \delta_{r-1}\right)$, which completes the proof of Theorem 2 .

4. The case of plane curves revisited. The reader might wonder why the bound for the error term in Theorem 2 in the case $r=2$ is not as sharp as the bounds for the error terms in (1) and (2). Following the proof of Theorems 1 and 2 above, it is clear that the quality of the upper bounds for the error terms provided by this method depends on the shape of the given region $\Omega$. Let us now see how one can recover the estimate (2), with exactly the same bound for the error term. We proceed as in the proof of Theorem 2 with $r=2$. The estimate (2) corresponds to the case $\mathbf{u}=\mathbf{0}$, but we take here a general $\mathbf{u}$ and choose a representative $\mathbf{v}=\left(v_{1}, v_{2}\right)$ of $\mathbf{u}$ in $\mathbb{R}^{2}$. 
The point now is that the region $A$ defined in the previous section does not need to be broken in small cubes since it can be written as a union of two parallelograms and a square: $A=A_{1} \cup A_{2} \cup A_{3}$, where

$$
\begin{aligned}
& A_{1}=\left\{\left(v_{1}+y_{1}, v_{2}+y_{2}\right) \in \mathbb{R}^{2}: 0 \leq y_{2}<1-\delta, 0 \leq y_{1}-y_{2}<\delta\right\}, \\
& A_{2}=\left\{\left(v_{1}+y_{1}, v_{2}+y_{2}\right) \in \mathbb{R}^{2}: 0 \leq y_{1}<1-\delta, 0 \leq y_{2}-y_{1}<\delta\right\}, \\
& A_{3}=\left\{\left(v_{1}+y_{1}, v_{2}+y_{2}\right) \in \mathbb{R}^{2}: 1-\delta \leq y_{1}, y_{2}<1\right\} .
\end{aligned}
$$

It follows that

$$
\left\{\mathbf{x} \in \mathcal{C}\left(\mathbf{F}_{p}\right): t(\mathbf{x}) \in \Omega\right\}=\Sigma_{1}+\Sigma_{2}+\Sigma_{3},
$$

where

$$
\begin{aligned}
& \Sigma_{1}=\sum_{\mathbf{x} \in \mathcal{C}\left(\mathbf{F}_{p}\right)} \chi_{[0,(1-\delta) p)}\left(x_{2}\right) \chi_{[0, \delta p)}\left(x_{1}-x_{2}\right), \\
& \Sigma_{2}=\sum_{\mathbf{x} \in \mathcal{C}\left(\mathbf{F}_{p}\right)} \chi_{[0,(1-\delta) p)}\left(x_{1}\right) \chi_{(0, \delta p)}\left(x_{2}-x_{1}\right), \\
& \Sigma_{3}=\sum_{\mathbf{x} \in \mathcal{C}\left(\mathbf{F}_{p}\right)} \chi_{[(1-\delta) p, p)}\left(x_{1}\right) \chi_{[(1-\delta) p, p)}\left(x_{2}\right) .
\end{aligned}
$$

Now each of the sums $\Sigma_{1}, \Sigma_{2}, \Sigma_{3}$ may be treated in the same way we estimated $N(\mathbf{J})$ in the proof of Theorem 1 . One obtains asymptotic results with square root upper bounds for the error terms as in (14). Putting all these together yields (2).

The above discussion shows that if the region $\Omega$ in Theorem 1 can be written as a union of $L$ nonoverlapping parallelepipeds in $\mathbb{T}^{r}$ then the upper bound for the error term in Theorem 1 can be replaced by $O_{r, d}\left(L p^{-1 / 2} \log ^{r} p\right)$. Thus in particular one has the following improved version of (5):

COROllary 1. Let $r \geq 2$ be an integer, $\boldsymbol{\delta}=\left(\delta_{1}, \ldots, \delta_{r-1}\right) \in \mathbb{R}^{r-1}$ with $0<\delta_{1}, \ldots, \delta_{r-1} \leq 1 / 2, p$ a prime number and $\mathcal{C}$ an irreducible curve of degree $d$ in $\mathbb{A}^{r}\left(\overline{\mathbf{F}}_{p}\right)$, defined over $\mathbf{F}_{p}$ and not contained in any hyperplane. Then

$$
\varrho_{r, p, \mathcal{C}, \delta}=2^{r-1} \delta_{1} \ldots \delta_{r-1}+O_{r, d}\left(p^{-1 / 2} \log ^{r} p\right) .
$$

\section{References}

[1] E. Bombieri, On exponential sums in finite fields, Amer. J. Math. 88 (1966), 71-105.

[2] H. Davenport, On a principle of Lipschitz, J. London Math. Soc. 26 (1951), 179-183.

[3] A. Weil, Sur les Courbes Algébriques et les Variétés qui s'en Déduisent, Hermann, Paris, 1948.

[4] W. Zhang, On the distribution of inverses modulo n, J. Number Theory 61 (1996), 301-310. 
[5] Z. Zheng, The distribution of zeros of an irreducible curve over a finite field, ibid. 59 (1996), 106-118.

Cristian Cobeli

Institute of Mathematics of the

Romanian Academy

P.O. Box 1-764

70700 Bucureşti, Romania

E-mail: ccobeli@stoilow.imar.ro
Alexandru Zaharescu Institute of Mathematics of the Romanian Academy P.O. Box 1-764 70700 Bucureşti, Romania

Department of Mathematics University of Illinois at Urbana-Champaign Altgeld Hall 1409 W. Green Street Urbana, IL 61801, U.S.A. E-mail: zaharesc@math.uiuc.edu

Received on 8.12.1999

and in revised form on 29.1.2001 